\title{
Concentration-dependent optical properties of TGA stabilized CdTe Quantum dots synthesized via the single injection hydrothermal method in the ambient environment
}

\author{
B. Jai Kumar, H.M. Mahesh* \\ Department of Electronic Science, Thin Film Solar Cell Lab, Bangalore University, Jnanabharathi Campus, Bangalore, 560056, India
}

\section{A R T I C L E I N F O}

\section{Article history:}

Received 16 January 2017

Received in revised form 13 February 2017

Accepted 13 February 2017

\section{Keywords:}

CdTe

TGA

Quantum dots

Single injection hydrothermal method

Ambient

Aqueous synthesis

\begin{abstract}
A B S T R A C T
Thioglycolic acid (TGA) stabilized aqueous CdTe Quantum dots (QDs) were synthesized using a facile, cost efficient Single Injection Hydrothermal (SIH) method. The complete preparation of precursors and growth of QDs was carried out in the ambient environment without inter gas protection. The Cadmium and Tellurium precursors were prepared from cadmium nitrate and elemental tellurium powder with sodium borohydride as reducing agent respectively. A systematic investigation was carried out in order to study the effect of $0.04 \mathrm{M}$ and $0.08 \mathrm{M}$ TGA concentration on ease synthesis, stability and size-tunable optical absorbance, bandgap, photoluminescence (PL) and Quantum yield (QY) of CdTe QDs. The Structure of QDs was verified by XRD and optical properties by absorbance and PL spectra. Experimental results revealed that the 0.08M TGA QDs possess good chemical and optical stability with high luminescence and decent QY, ready to use in optoelectronics, photovoltaic and biological application.
\end{abstract}

() 2017 Elsevier Ltd. All rights reserved.

\section{Introduction}

Quantum dots are the semiconductor nanocrystal whose size is lesser than Bohr's radius of that material, leading to profound Quantum confinement effect. This leads to remarkable optical and electrical properties such as size-tunable absorbance, tailored band gap, multiple exciton generation, high photoluminescence and superior photostability. These unique properties give rise to immense applications in optoelectronics, photovoltaic and biological instruments [1-10]. Among many QDs, Cadmium Telluride (CdTe) has potential advantages of direct band gap which could be tuned from 3.9 to $1.5 \mathrm{eV}$ covering most of the solar spectrum and significant absorption coefficient giving rise to the photovoltaic applications, a broad range of PL emission windows and near IR activities is suitable for biological and photoelectron devices [1-10]. Hence, synthesis and processing of high-quality nanocrystal with mono-disperse, size-tunable and high QYs is obviously most competitive research area.

Abbreviations: SIH, Single Injection Hydrothermal; CdTe, Cadmium Telluride; QDs, Quantum Dots; $\left.\mathrm{Cd}_{(\mathrm{NO}}\right)_{2}, \mathrm{Cadmium}$ nitrate; Te, Tellurium; NaBH 4 , Sodium Borohydride; TGA, Thioglycolic Acid; $\mathrm{NaOH}$, Sodium hydroxide; $\mathrm{NaHTe}$, Sodium Hydrogen Telluride; $\mathrm{Al}_{2} \mathrm{Te}_{3}$, Aluminum telluride; XRD, X-ray Diffraction; PL, Photoluminescence; FWHM, Full width at half maximum; QY, Quantum yield.

* Corresponding author.

E-mail address: hm_mahesh@rediffmail.com (H.M. Mahesh). 
Among various protocols developed to synthesize different sized, shaped QDs in past few decades, 'colloid synthesis' is significant. This is also referred as 'arrested precipitation of QDs-ligand complex' in which inorganic core is stabilized by a layer of organic ligand shell. The properties of the QDs not only dependents on the inorganic core material but also on the organic ligand shell chosen; as the stability, nature and interaction of QDs mainly depend on the binding between ligand and surface atoms of core material [11-16]. This colloidal QDs synthesis is further classified as organometallic and aqueous synthesis. The former exhibits the strength of high QY with mono-sized QDs, but also some weaknesses like they required sophisticated and expensive equipment and laborites [10,16,17]. Further, the syntheses take place at high temperature and QDs should be pretreated to make it biocompatible, this process reduces the QY. Whereas, the later one is carried out at low temperature and are water soluble, hence biocompatible.

Aqueous synthesis can be further divided on the basis of synthesis method and Te precursor used. Among several methods microwave irradiation, electrochemical, illumination, ultrasound, and hydrothermal route [18-24] are prominent. All through all these techniques are efficient hydrothermal route is the simplest with least instrument required. Among Te Precursor $\mathrm{Al}_{2} \mathrm{Te}_{3}$ [25-28], $\mathrm{NaTeO}_{3}, \mathrm{TeO}_{2}$ [23,24], Te (as an electrode) and elemental Te powder [20,29-31] are the important sources, all sources are expensive except elemental tellurium and also requires a complex instrument to handle them. But, Te precursor using Te is prepared in an inert atmosphere to prevent oxidation of Te, this increases the cost of reaction. Hence it was felt worth to go through the hydrothermal synthesis procedure and develop a simple, convenient and cost-effective method to produce mono-sized QDs with good QY. For achieving this, selecting of the ligand also plays a very vital role, which impact on quality, optical and electrical properties, solubility and interaction of QDs with other materials [11-16]. Therefore, TGA was chosen this is a short, straight chained, water soluble stabilizing agent, with required steric hindrance helping to prevent aggregations of QDs and to passivate QDs surface, improving PL emission and interaction with the environment [31-33]. Hence, in the present investigation, we have developed a facile single injection hydrothermal method to synthesize TGA-capped CdTe QDs using element Te powder as Te precursor, preventing oxidation of Te in the complete ambient environment. Further TGA concentration-dependent chemical reaction and optical properties are studied in detail and analyzed.

\section{Experimental details}

\subsection{Materials}

Cadmium nitrate tetrahydrate $\left(\mathrm{Cd}\left(\mathrm{NO}_{3}\right)_{2} 4 \mathrm{H}_{2} \mathrm{O}, 98 \%\right.$, Sigma-Aldrich) as cadmium precursor, Tellurium powder (Te, -200 mesh, 99.8\%, Aldrich) and Sodium Borohydride $\left(\mathrm{NaBH}_{4}, 98 \%\right.$, Aldrich) as Te source and reducing agents respectively, Thioglycolic acid (TGA, $\mathrm{C}_{2} \mathrm{H}_{4} \mathrm{O}_{2} \mathrm{~S}, 99 \%$, Sd fine chem. Limited) as stabilizer and Sodium hydroxide ( $\mathrm{NaOH}$, Spectrochem) to maintain $\mathrm{pH}$, solvent - distilled water were used for the synthesis of TGA-capped CdTe QDs, all chemical were used as procured without further purification.

\subsection{Synthesis of TGA-capped CdTe QDs}

TGA-capped CdTe QDs were synthesized in two different concentration of TGA to Cd ratio, using SIH method in the complete ambient atmosphere. The detailed synthesis procedure is given in our previews work MPA-capped CdTe QDs [3] and a brief synthesis protocol is described in this paper. The synthesis process consists of three parts. First: preparation of TGAcapped Cd precursor. Second: synthesis of Te precursor, NaHTe preventing oxidation of Te. Finally, the growth of TGA-capped CdTe QDs. TGA-capped Cd precursor is prepared in two different ratio of TGA to Cd that is 1:1 and 2:1 ratio, with Molarity of 0.04M: $0.04 \mathrm{M}$ and $0.08 \mathrm{M}$ : $0.04 \mathrm{M}$ respectively for TGA and Cd.

Cd precursor: To start with, $5 \mathrm{ml}$ of $0.04 \mathrm{M}$ TGA solution is added to $10 \mathrm{ml}$ of distilled water and stirred for 10 mintues. To this $5 \mathrm{ml}$ of $0.04 \mathrm{M} \mathrm{Cd}$ solution is added and stirred for 10 mintues. Followed by adjusting the $\mathrm{pH}$ of the solution to 10 using $1 \mathrm{M}$ $\mathrm{NaOH}$. The $\mathrm{pH}$ of the solution changes for acidic to basic, with a visible change in color from a clear solution to cloudy white (Equation (1)) and back to clear solution (Equation (2)). This clear Cd precursor is stirred until NaHTe is ready.

$\mathrm{Cd}\left(\mathrm{NO}_{3}\right)_{2}+2 \mathrm{NaOH} \rightarrow \mathrm{Cd}(\mathrm{OH})_{2}+2 \mathrm{NaNO}_{3}$

$\mathrm{Cd}(\mathrm{OH})_{2}+4 \mathrm{NaOH} \rightarrow \mathrm{Cd}^{2+}+$ (other bi-products)

Te precursor: NaHTe is prepared by reducing elemental Te powder using $\mathrm{NaBH}_{4}$ as reducing agent, Te to $\mathrm{NaBH}_{4}$ were taken in ratio 1:2.5 respectively, this high ratio of $\mathrm{NaBH}_{4}$ help in fast reduction of Te power. First, $5 \mathrm{ml}$ of $0.2 \mathrm{M} \mathrm{NaBH}_{4}$ is taken in a conical flask to this $5 \mathrm{ml}$ of distilled water and $0.08 \mathrm{M}$ equivalent Te powder is added. The conical flask is capped tightly, and then few small vent holes are made for $\mathrm{H}_{2}$ gas liberation. The flask is well shaken and kept in preheated water bath $\left(90^{\circ} \mathrm{C}\right)$. Here oxidation of Te in ambient is prevented by insult generated $\mathrm{H}_{2}$ gas, which intern reduces the oxygen inside the conical flask. Further, NaHTe formation can be seen from the change in color from dark black solution to clear solution with the liberation of $\mathrm{H}_{2}$ gas Equation (3) [3]. Then from a clear solution to dark pink color, at this stage $2 \mathrm{ml}$ (of $10 \mathrm{ml}$ ) NaHTe is injected to Cd precursor.

$4 \mathrm{NaBH}_{4}+2 \mathrm{Te}+4 \mathrm{H}_{2} \mathrm{O} \rightarrow 2 \mathrm{NaHTe}+11 \mathrm{H}_{2} \uparrow+2 \mathrm{NaBO}_{2}$ 
The Cd solution turns to a golden color indicating the formation of CdTe monomers. This solution is stirred at room temperature for $10 \mathrm{mintues}$ and then at $90^{\circ} \mathrm{C}$. Finally, TGA-capped CdTe QDs aliquots are collected at different intervals of reflux time and analyzed. Similarly, TGA-capped CdTe QDs with TGA Molarity of 0.08M was prepared, keeping all other parameters same. The increase of TGA concentration helps in balancing the pH easily and also prevents the formation of white turbulences while heated. However, this white turbulence in 1:1 ratio of TGA: Cd can be retrieved by reducing the pH of the solution back to $10 \mathrm{pH}$. The higher $\mathrm{pH}$ of solution helps in fast growth of QDs and hence in this experiment the pH was maintained at 10. The growth of TGA-capped CdTe QDs is depicted using Equations (4) and (5) and its schematic is as shown in Fig. 1.

$\mathrm{Cd}^{2+}-\mathrm{SR}+\mathrm{Te}^{2-} \rightarrow \mathrm{RS}-\mathrm{CdTe}$

n TGA-CdTe $\rightarrow$ (TGA-CdTe)n

From the synthesized solution, QDs powder was extracted by precipitating using acetone. Further purified using methanol, to remove the un-reacted chemicals with repeated wash and the final precipitate was centrifuged and vacuum dried. This purified QDs powder was used for other characterization.

\subsection{Instruments used for characterizations}

Synthesized CdTe QDs were characterized for structural and optical properties. QDs structure was studied by XRD (Rigaku smart lab XRD system) in $2 \Theta$ range $20^{\circ}-70^{\circ}$ with a step size of $0.002^{\circ}$. Optical absorbance and transmittance of CdTe QDs solution were measured using UV-Vis-NIR spectrophotometer (Ocean optics USB 4000) and PL by LabRAM HR spectrometer and quantum yield was calculated with respect to Rhodamine B (considering QY $=100 \%$ ) with an excitation wavelength of $430 \mathrm{~nm}$ and absorbance was kept below 0.1 for all the samples.

\section{Results and discussion}

CdTe QDs growth process can be explained in three stages. In the first stage $\mathrm{Cd}^{2+}$ and $\mathrm{Te}^{2-}$ monomers are synthesized. When these two monomers come in contact, result in the second stage that is fast nucleation stage where the ionic bond between $\mathrm{Cd}^{2+}$ and $\mathrm{Te}^{2-}$ take place resulting in a large number of CdTe monomers. Also in this stage, QDs size increases in fast phase as there is plenty of $\mathrm{Cd}$ and Te monomers. As $\mathrm{Cd}^{2+}$ and $\mathrm{Te}^{2-}$ concentration deplete, the third stage Ostwald ripening (OR) stage beings. At this stage, small QDs crystals dissociate and get deposited on the surface of large sized QDs facilitating the growth of larger sized QDs (as depicted in Fig. 2). In OR stage the small sized QDs have greater solubility than the large sized QDs, due to high Gibbs energy, which intern results in decomposition of same sized QDs. Further, this OR stage results in stable crystal with reduced total Gibbs energy. But, the QDs size grows in slow phase, increasing the reaction time to obtain large-sized QDs.

\subsection{XRD analysis}

Powder XRD of the synthesized TGA CdTe Quantum dots is depicted in Fig. 3. The diffraction peaks match with the JCPDS card number 75-2086 of CdTe and $2 \theta$ peaks at 24is assigned to 111 planes. The inter-planer spacing (d) between the atoms is determined using Bragg's Law [34] 2d $\sin \theta=\mathrm{n} \lambda$; where $\mathrm{n}=1$ and $\lambda=1.5418 \AA$ wavelength of Cuk $\alpha$ at $2 \theta=24^{\circ}$ and $\mathrm{d}$ was found to be $3.7 \AA$. Further the lattice spacing (a) were determined using equation for cubic structure; $a^{2}=\left(h^{2}+k^{2}+l^{2}\right) d^{2}$ where $\mathrm{h} \mathrm{k} \mathrm{l}$ are the lattice plane values, and in the present state $h k l$ is 111 and the calculated a $=\sim 6.42 \AA$ which agrees with JCPDS card number 75-2086. There is no trace of oxidation of Te in diffraction pattern and hence the ambient synthesis protocol could be used for large scale synthesis.

\subsection{Optical properties}

The optical property is one of the significant properties used to determine the optical band gap and size of QDs. Optical absorbance and transmittance directly show the range of wavelength QDs absorb. Fig. 4(a) show the absorption spectra of the

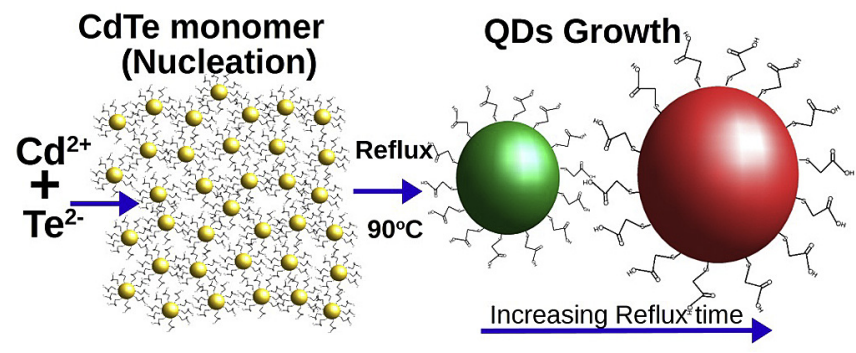

Fig. 1. The schematic diagram showing the synthesis process of the TGA capped CdTe QDs as reflux time increasing. 


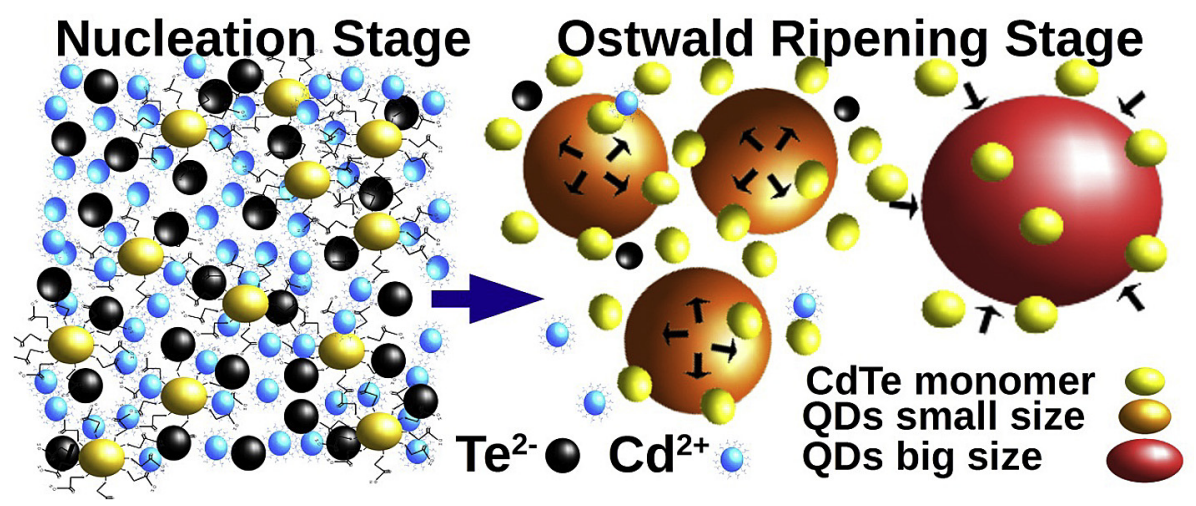

Fig. 2. The schematic diagram showing the Nucleation and Ostwald Ripening Stage of the growth process.
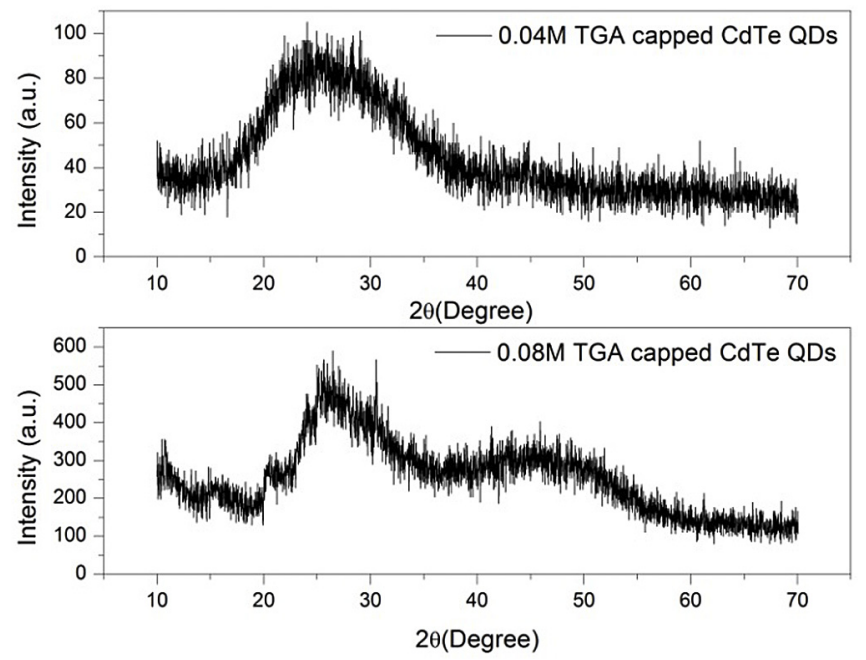

Fig. 3. X-ray powder diffraction patterns of TGA-capped CdTe QDs (0.04M TGA QDs: Refluxed 150 min, $0.08 \mathrm{M}$ TGA QDs: Refluxed 500 min).
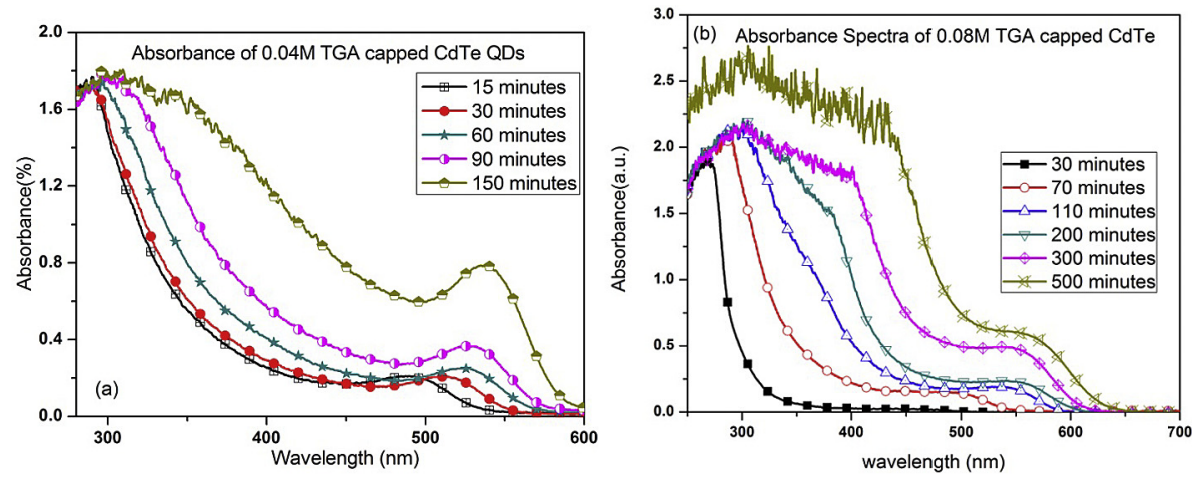

Fig. 4. Absorbance spectra of (a) 0.04M and (b) 0.08M TGA-capped CdTe Quantum dots synthesized using the single injection hydrothermal method.

0.04M TGA concentration CdTe QDs aliquots collected at 15, 30, 60, 90 and 150 min. The absorption spectra show wellresolved absorption peaks indicating first allowed electronic transition from 1s holes to 1s electron band state (e1h1). The absorption peaks have blue shifted with respect to bulk CdTe absorption peak due to quantum confinement effect. The absorption peak of aliquot collected at $15 \mathrm{~min}$ reflux time as red shifted from $490 \mathrm{~nm}$ to $540 \mathrm{~nm}$ as reflux time as increased to 150 min. Implying that the QDs as grown with the increase in reflux time. The high intensity of absorption peaks with narrow 
full-width half maximum depicts that, the size distribution of synthesized QDs are highly uniform. In addition, the absorption spectrum confirms that the synthesized QDs absorb a wide range of incident energy from $200 \mathrm{~nm}$ to $600 \mathrm{~nm}$.

Similarly Fig. 4(b) shows the absorption spectra of 0.08M QDs aliquots collected at 30, 70, 110, 200, 300 and 500 min reflux time. The trend is similar to 0.04M QDs, with absorption peaks blue shifted with respect to bulk CdTe absorption peak and QDs collected at 500 min have red shifted with respect to 30 min QDs showing the QDs size has grown as reflux time as increased. However, absorption range as increased compared to 0.04M QDs to $650 \mathrm{~nm}$. Further, the shifts in absorption peak position show a fast shift at the initial stage of the reaction and slow shift later on. This is mainly due to the growth process, as at early stage $\mathrm{Cd}^{2+}$ and $\mathrm{Te}^{2-}$ ions are more resulting in fast nucleation and as they deplete QDs growth rate reduces and here after QDs growth take place due to Ostwald ripening process. This QDs growth process is true to both concentrations of TGA QDs. Even when the slow growth rate in $0.08 \mathrm{M}$ can be seen compared to $0.04 \mathrm{M}$ TGA-capped QDs, due to strong binding of TGA on to Cadmium surface. But, 0.08M QDs were stable in the solution whereas 0.04M QDs were not and practically it's difficult to maintain $\mathrm{pH}$ constant in $0.04 \mathrm{M}$ concentration than in $0.08 \mathrm{M}$ concentration QDs.

The transmittance spectra of sample collected at different reflux time is shown in Fig. 5(a) and (b) corresponding to 0.04M and 0.08M QDs. Both transmittance spectra show a transmission edge has red shifted as reflux time as increased due to increases in QDs size. Further, the spectrums show two transmission edges, depicting that the QDs are strongly confined resulting in clear multiple bands, therefore QDs results in multiple band gap energy. The synthesized QDs show transmittance above $90 \%$ above $550 \mathrm{~nm}$ wavelength for aliquot collected at 15 min reflux time and this as red shifted to above $600 \mathrm{~nm}$ wavelength for aliquot collected at $150 \mathrm{~min}$.

CdTe QDs of 0.04M concentration show high energy transmittance edge shifting from $426 \mathrm{~nm}$ to $493 \mathrm{~nm}$, correspondingly low energy transmittance edge as red shifted from $535 \mathrm{~nm}$ to $600 \mathrm{~nm}$, as reflux time increased from 15 to $150 \mathrm{~min}$. In similar manner high energy transmittance edge shift from 390 to $515 \mathrm{~nm}$ and lower edge from 509 to $654 \mathrm{~nm}$ for reflux time 30 to $500 \mathrm{~min}$ in 0.08M QDs. Also, high energy transmittance edge intensity has decreased as reflux time as increased in both spectra, clearly showing the incident photons are absorbed faster with increases in QDs size. The maximum transmittance above $550 \mathrm{~nm}$ in $0.04 \mathrm{M}$ QDs as decreases from $100 \%$ to $90 \%$ as reflux time as increased from 15 to 150 min, this effect was mainly due to increases in white turbulences appearing in $0.04 \mathrm{M}$ QDs solution as reflux time increased. This turbulence was clear indication that 1:1 ratio taken for TGA: Cd was insufficient to bind all Cd by TGA, whereas in 0.08M QDs the maximum transmittance is almost constant for all aliquot implying that 2:1 ratio of TGA to $\mathrm{Cd}$ was an effective ratio to bind $\mathrm{Cd}^{2+} / \mathrm{CdTe}^{2}$ monomers with TGA.

\subsection{Photoluminescence properties}

Photoluminescence spectra of TGA-capped CdTe QDs are shown in Fig. 6(a) and (b) respectively for 0.04M and 0.08 TGA concentrations. For 0.04M concentration PL peak as shifter from 521.5 to $563.4 \mathrm{~nm}$, corresponding absorption peaks were 490 and $540 \mathrm{~nm}$ respectively for aliquots collected at 15 to $150 \mathrm{~min}$ (details in Table 1 ). Similarly, for $0.08 \mathrm{M}$, PL peaks as red shifted from 517.8 to $613.8 \mathrm{~nm}$, corresponding absorption peaks were 462 and $562 \mathrm{~nm}$, respectively for aliquots collected at 30 to 500 min (details in Table 2). The red shift in PL peaks is clearly due to the growth of QDs, also the PL peak shift with respect to reflux time proves that QDs size grows in fast phase in nucleation stage and then slow growth in OR process. Fig. 6(c) shows the second PL emission of 0.08M CdTe QDs, all sized QDs shows peak at $858 \mathrm{~nm}$, which is due to the emission from second inter band transition and this also confirms the strong confinement of the synthesized QDs.

The full-width half-maximum (FWHM) of the PL peak as subsequently increased from 36 to $45 \mathrm{~nm}$ correspondingly for 15-150min reflux time in 0.04M QDs. In 0.08M QDs, FWHM has increased from 38.6 to $65 \mathrm{~nm}$ respectively for 30-500 min refluxed QDs. This increase in FWHM of the PL peaks is due to change in QDs size distribution. Size distribution of QDs depends on the synthesis process, at the initial stage of the synthesis, fast nucleation takes places due to which size
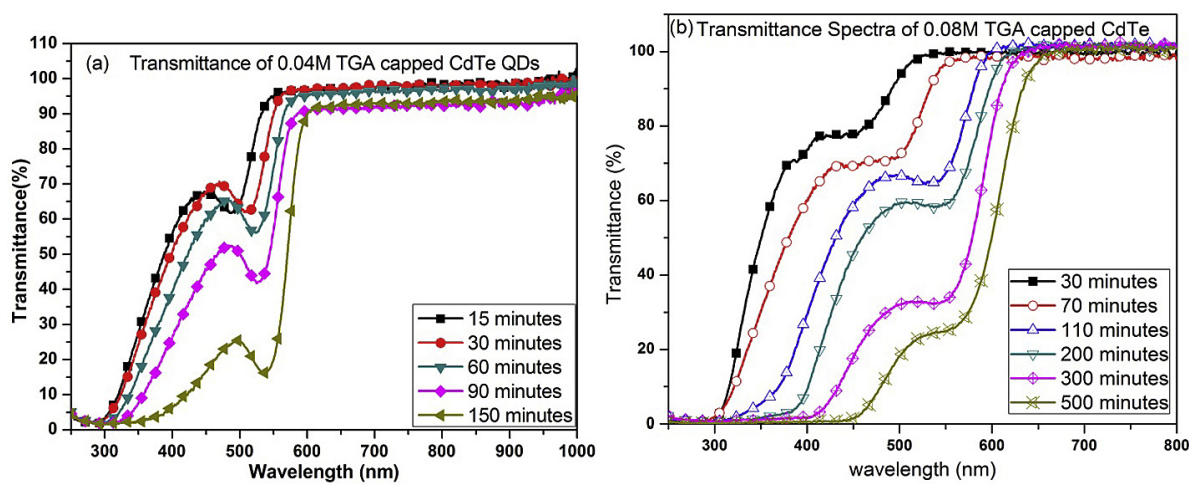

Fig. 5. Transmittance spectra of (a) $0.04 \mathrm{M}$ and (b) $0.08 \mathrm{M}$ TGA-capped CdTe Quantum dots synthesized using the single injection hydrothermal method. 

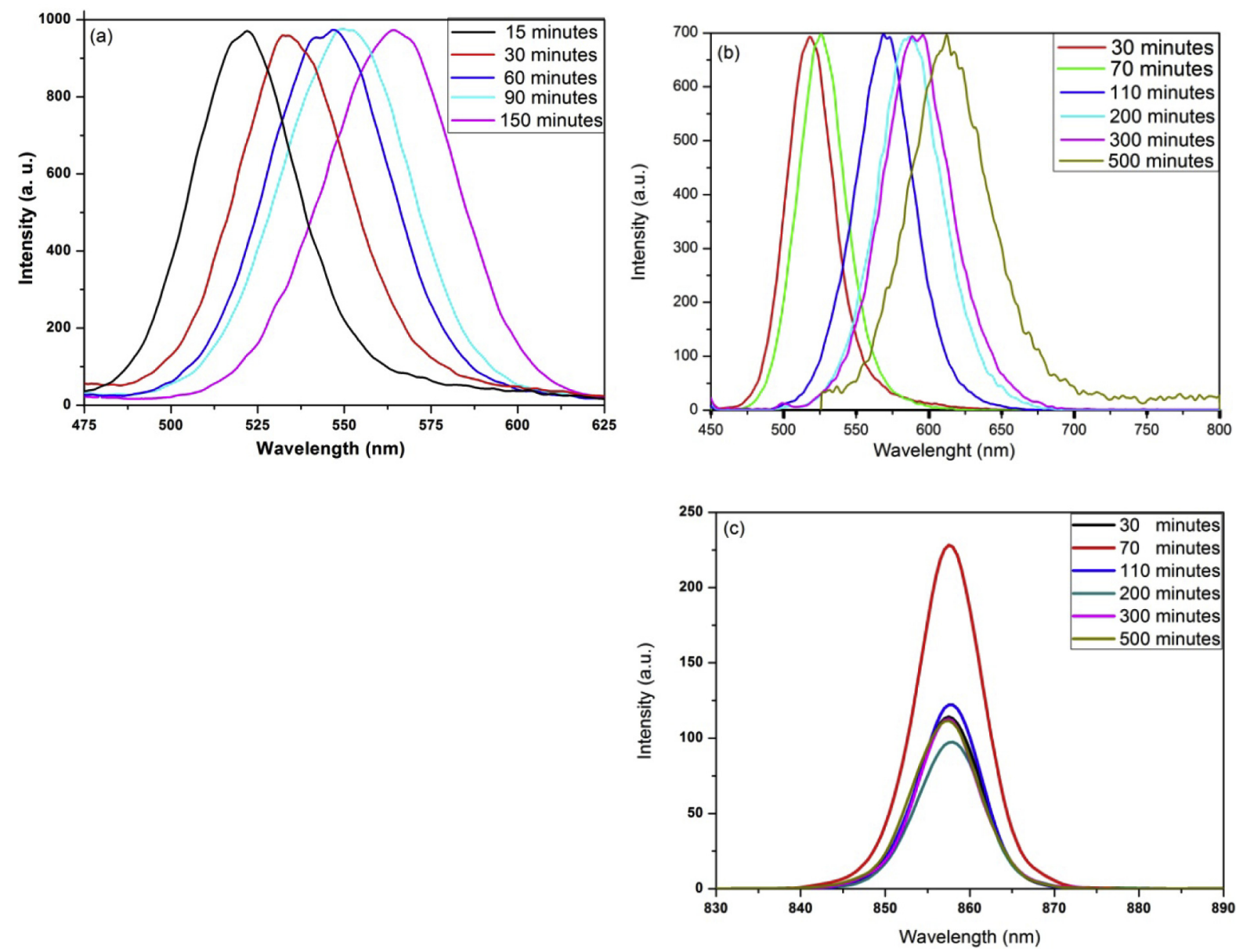

Fig. 6. Photoluminescence spectra of (a) 0.04M, (b) 0.08M TGA-capped CdTe QDs (c) second PL emission peak of 0.08M TGA-capped CdTe QDs.

Table 1

Absorption peak, PL peak, FWHM and QY of 0.04M TGA-capped CdTe QDs.

\begin{tabular}{|c|c|c|c|c|c|c|}
\hline SI. No & Reflux time (in minutes) & Absorption Peak (nm) A & Photoluminescence peak (nm) P & FWHM (nm) & QY (in \%) & Diff P-A \\
\hline 1 & 15 & 490.4 & 521.5 & 35.8 & 0.67 & 31.1 \\
\hline 2 & 30 & 512.8 & 535.4 & 38.5 & 1.42 & 22.6 \\
\hline 3 & 60 & 525.0 & 545.6 & 41.7 & 1.96 & 20.6 \\
\hline 4 & 90 & 529.8 & 550.3 & 43.6 & 2.76 & 20.5 \\
\hline 5 & 150 & 539.6 & 563.4 & 45.1 & 2.39 & 23.8 \\
\hline
\end{tabular}

Table 2

Absorption peak, PL peak, FWHM and QY of 0.08M TGA-capped CdTe QDs.

\begin{tabular}{|c|c|c|c|c|c|c|}
\hline SI. No & Reflux time (in minutes) & Absorption Peak (nm) A & Photoluminescence peak (nm) P & FWHM (nm) & QY (in \%) & Diff P-A \\
\hline 1 & 30 & 462.2 & 517.8 & 38.6 & 5.48 & 55.6 \\
\hline 2 & 70 & 498.8 & 526.3 & 39.9 & 22.05 & 27.5 \\
\hline 3 & 110 & 540 & 569.9 & 49.7 & 17.19 & 29.9 \\
\hline 4 & 200 & 550.7 & 586.3 & 54.4 & 7.23 & 35.6 \\
\hline 5 & 300 & 552.21 & 591.5 & 57.2 & 4.81 & 39.29 \\
\hline 6 & 500 & 562 & 613.5 & 64.8 & 1.69 & 51.5 \\
\hline
\end{tabular}

distribution of QDs is narrow (less) and as a monomer of $\mathrm{Cd}^{2+}$ and $\mathrm{Te}^{2-}$ is depleting the OR process increases resulting in broad size distribution and hence increases in FWHM.

The absorption peak, PL peak, FWHM, QY and shift in PL peak with respect to absorption peak for QDs collected at different reflux times, respectively for 0.04M-0.08M TGA-capped CdTe QDs have been presented in Tables 1 and 2. Quantum yield was 
calculated using relative measuring method comparing the intensity of the standard sample with an unknown sample, QY of the QDs was obtained comparing it to the standard Rhodamine B sample using the equation given below.

$$
Q Y_{x}=Q Y_{s t}\left(\frac{A_{s t}}{A_{x}}\right)\left(\frac{F_{x}}{F_{s t}}\right)\left(\frac{n_{x}^{2}}{n_{s t}^{2}}\right)
$$

where, $Q Y_{s t}$ and $Q Y_{x}$ is quantum yield, $A_{s t}$ and $A_{x}$ is absorbance at excitation wavelength which was kept below 0.1 for all the sample, $F_{x}$ and $F_{s t}$ is area of emission spectrum, $n_{x}$ and $n_{s t}$ is refractive index of the solvent (solvent used were ethanol with $n_{s t}=1.3661$ for standard and water with $n_{x}=1.3329$ for QDs) used of standard sample (st) and unknown (x-QDs in current case) respectively. To a certain point, $\mathrm{QY}$ as increased and again decreased. This variation is mainly related to growth process and hence related to surface defect and size distribution of QDs. At fast nucleation stage, surface defects in QDs are high. Hence, leads to shifting in PL peak corresponding to absorption peak, as PL emission takes place from defects state of QDs. Decrease in QY is also due to the initial stage of formation of CdTe monomers, where the monomer is high compared to QDs grown resulting in less chemical yield and intern less QY. As the reflux time increases, the surface defects of nanocrystal decreases due to annealing effect, resulting in less shift in PL peak with respect to absorption peak, meanwhile nucleation process reduces due to a reduction in monomer concentration and OR process begins. Hence at equilibrium of nucleation and OR process with reduced surface defects, QYs reaches the highest. One's OR process overtakes nucleation process it again results in increases in the size distribution of QDs, which intern results in a higher shift in the PL peak positions, as multiple sized QDs emit in different position resulting in broadening in PL peak and hence QY also reduces. Furthermore, even when all explanation mentioned earlier holds good for both the concentration, the QY of $0.04 \mathrm{M}$ is very less compared to $0.08 \mathrm{M}$ QDs, this was due to less stability of the formed CdTe monomer due to lack of TGA concentration to bind them completely. These QDs with decent QY are most suitable for many PL applications.

\subsection{Optical band gap TGA-capped CdTe QDs}

Band gap is one of an important parameter which shows the effect of confinement. The optical band gap energy is obtained from Tauc's plot [35] (Equation (6))

$$
\alpha h v=A\left(h v-E_{g}\right)^{m}
$$

where ' $A$ ' is optical constant, ' $h v$ ' is the incident photon energy, ' $E_{g}$ ' is optical band gap energy, $\mathrm{m}$ is a constant which depends on the type of transition and it takes values, 1/2,3/2, 2, 3 respectively for direct allowed, direct forbidden, indirect allowed and indirect forbidden transitions. ' $\alpha$ ' is the optical absorption coefficient determined using Equation (7).

$$
\alpha=\frac{\operatorname{Ln}\left(\frac{1}{T}\right)}{t}
$$

where $\mathrm{T}$ is transmittance and $\mathrm{t}$ is the path length in which incident light pass in the solution. For CdTe QDs $\mathrm{m}=1 / 2$ as CdTe is direct band transition material. Optical band gap $\left(\mathrm{E}_{\mathrm{g}}\right)$ is defined as the minimum energy required to excite electrons from the valence band to conduction band of the materials. To determine $\mathrm{E}_{\mathrm{g}}$, the linear region of the Tauc's plot $\left\{(\alpha h v)^{2} \mathrm{vs} . h v\right\}$ is extrapolated to meet $\mathrm{x}$ axis $(h v)$ that is $(\alpha h v)^{2}=0$ or $h v=E_{g}$. In bulk materials, the band gap is equal to the energy separation between valence and conduction band edges and hence a single band gap. However in QDs due to quantum confinement effect the valence and conduction band had been sub divided and results in distinct energy levels and hence multiple band gaps can be found in Tauc's plot.

Fig. 7(a) depicts the high energy band gap of 0.04M QDs and it as red shifted from 3.72 to 2.69eV, correspondingly Fig. 7(b) show the low energy band gap of 0.04M QDs and it as decreased from 2.36 to $2.11 \mathrm{eV}$ respectively for 15-150 min QDs aliquots. Similarly Fig. 8(a, b) shows band gap of 0.08M QDs, the high energy band gap as decreased from 3.8 to 2.6eV, with low energy band gap from 2.5 to $1.97 \mathrm{eV}$ respectively for 30 to 500 mintues QDs samples. Band gap achieved in 0.08M QDs is less than $0.04 \mathrm{M}$ QDs, indicating that the QDs size as grown more in $0.08 \mathrm{M}$ concentration, intern reduced quantum confinement energy hence the band gap is moving toward bulk band gap as shown in Fig. 9.

\subsection{Calculation of TGA-capped CdTe QDs size}

The size of the CdTe QDs is obtained using Brus equation (Equation (8)) [1], which is rearranged (Equation (9)) to express the size of the QDs in terms of the bandgap.

$$
\mathrm{E}_{\mathrm{g}}^{\mathrm{QD}}=\mathrm{E}_{\mathrm{g}}^{\text {Bulk }}+\frac{\mathrm{h}^{2}}{8 \mathrm{r}^{2}}\left[\frac{1}{\mathrm{~m}_{\mathrm{e}}^{*}}+\frac{1}{\mathrm{~m}_{\mathrm{h}}^{*}}\right]-\frac{1.786 \mathrm{e}^{2}}{4 \pi \in_{\mathrm{o}} \in_{\mathrm{r}} \mathrm{r}}
$$



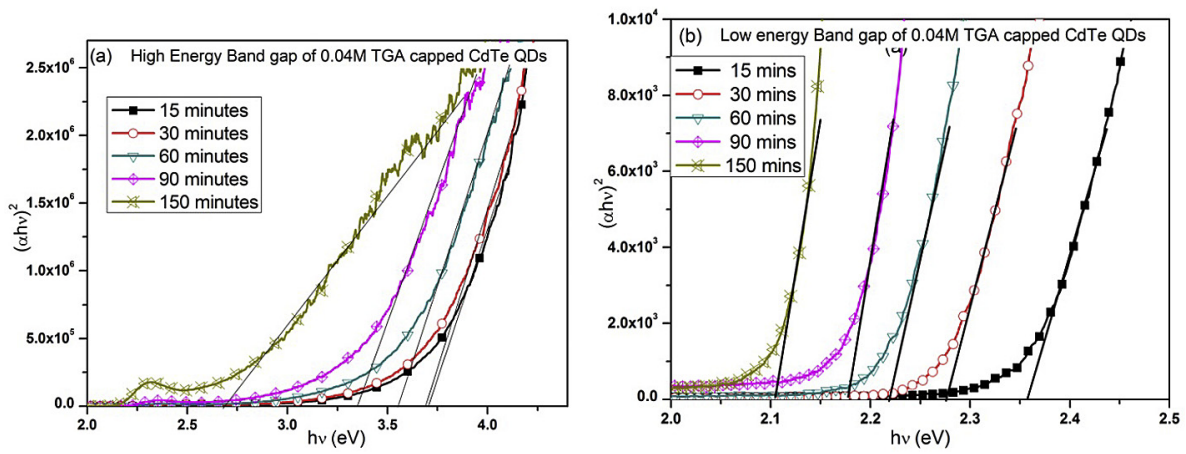

Fig. 7. (a) high energy band gap and (b) low energy band gap of 0.04M TGA-capped CdTe QDs.
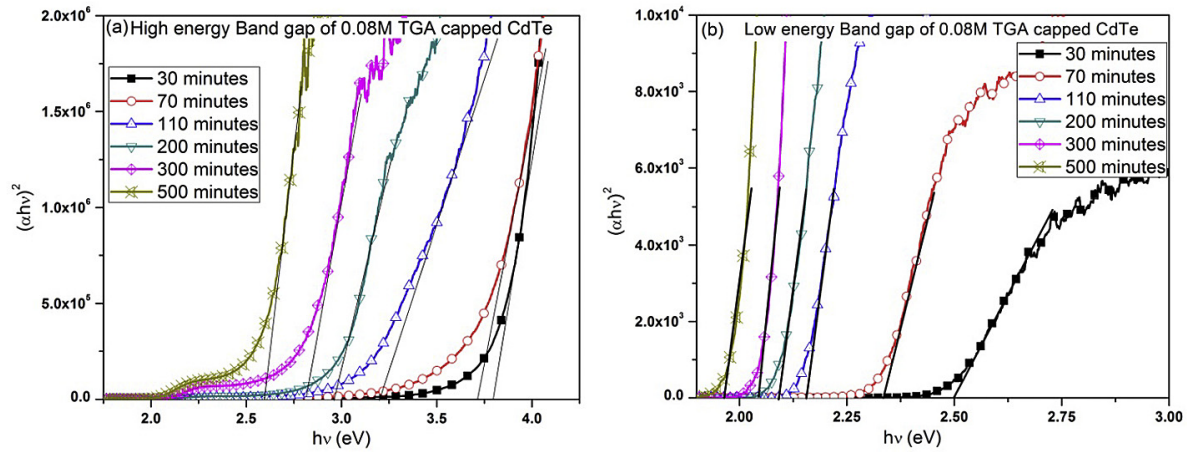

Fig. 8. (a) high energy band gap and (b) low energy band gap of 0.08M TGA-capped CdTe QDs.

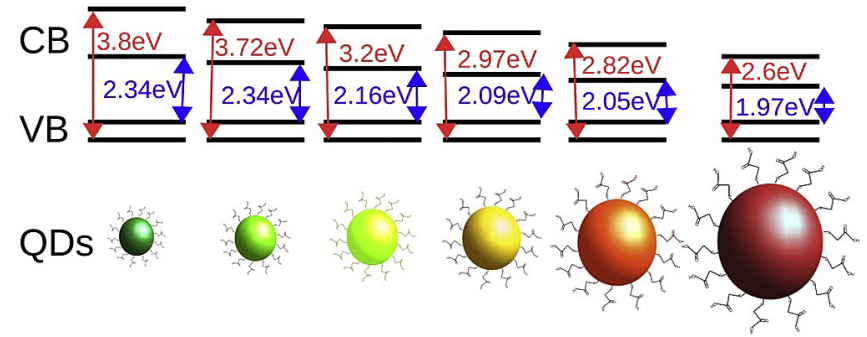

Fig. 9. The schematic diagram showing the multiple band gaps with increasing QDs size.

$$
\mathrm{r}=\frac{-\left[\frac{1.786 \mathrm{e}^{2}}{4 \pi \varepsilon_{0} \varepsilon_{\mathrm{r}}}\right]+\sqrt{\left[\frac{1.786 \mathrm{e}^{2}}{4 \pi \varepsilon_{0} \varepsilon_{\mathrm{r}}}\right]^{2}+\left[\mathrm{E}_{\mathrm{g}}^{\mathrm{QD}}-\mathrm{E}_{\mathrm{g}}^{\mathrm{Bulk}}\right] \frac{\mathrm{h}^{2}}{2}\left[\frac{1}{\mathrm{~m}_{\mathrm{e}}^{*}}+\frac{1}{\mathrm{~m}_{\mathrm{h}}^{*}}\right]}}{2\left[\mathrm{E}_{\mathrm{g}}^{\mathrm{QD}}-\mathrm{E}_{\mathrm{g}}^{\mathrm{Bulk}}\right]}
$$

where: $r$ is Radius of QDs $(\mathrm{nm}), \mathrm{E}_{\mathrm{g}}^{\mathrm{QD}}$ and $\mathrm{E}_{\mathrm{g}}^{\text {Bulk }}$ band gap (eV) of QDs and bulk semiconductor respectively, $\in_{\mathrm{o}}$ and $\in_{\mathrm{r}}$ absolute and relative permittivity respectively, $\mathrm{m}_{\mathrm{e}}^{*}$ and $\mathrm{m}_{\mathrm{h}}^{*}$ is an Effective mass of electron and hole respectively, for CdTe bulk band gap is $1.475 \mathrm{eV}, \Theta \mathrm{r}=7.1, \mathrm{~m}_{\mathrm{e}}^{*}=0.11 \mathrm{mo}, \mathrm{m}_{\mathrm{h}}^{*}=0.35 \mathrm{mo}$, where $\mathrm{mo}$ is an absolute mass of the electron.

The variation of the band gap and QDs size (Calculated for low energy band gap) vs. reflux time is shown in Fig. 10(a) and b respectively for 0.04 and $0.08 \mathrm{M}$ QDs. The size obtained for $0.04 \mathrm{M}$ varied from 4.2 to $4.92 \mathrm{~nm}$ and similarly for $0.08 \mathrm{M}$ size varied from 3.92 to $5.54 \mathrm{~nm}$. The actual size of the small crystal is comparatively less than size calculated for Brus equation. The largest size obtained is still under Bohr exciton radius $\left(\mathrm{a}_{\mathrm{ex}}\right)$ of $\mathrm{CdTe}\left(\mathrm{a}_{\mathrm{ex}}=\sim 7.3 \mathrm{~nm}\right)$ [2], hence all the QDs are well under strong quantum confinement regime $\left(\mathrm{R} \ll \mathrm{a}_{\mathrm{ex}}\right)$. The obtained $\mathrm{QDs}$ radius was used in brus equation and the next allowed transition band energy was calculated using Equation (10) and compared with the measured band gap. 

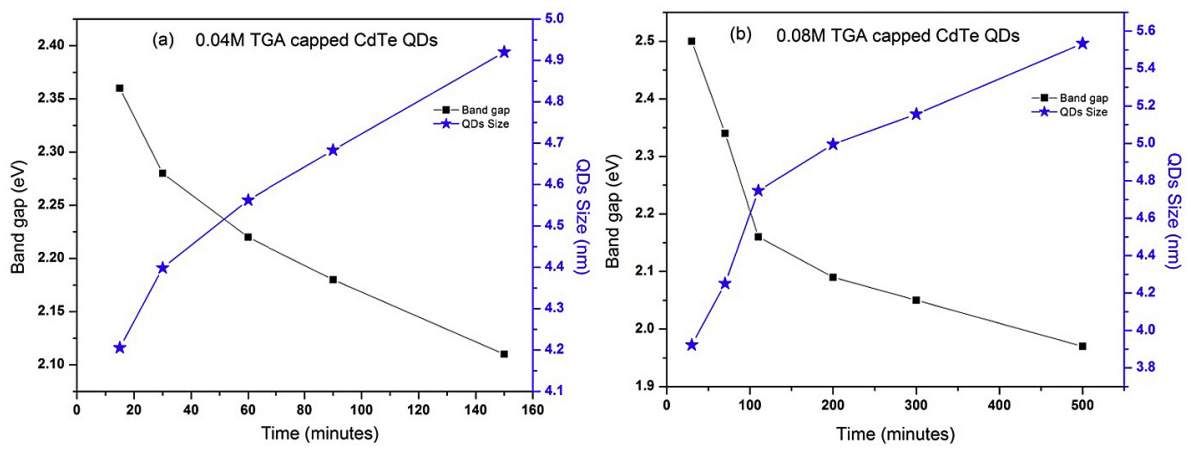

Fig. 10. Variation of Band gap and size of QDs with respect to reflux time of (a) 0.04M and (b) 0.08M TGA-capped CdTe QDs.

$$
\mathrm{E}_{\mathrm{g}}^{\mathrm{QD}}=\mathrm{E}_{\mathrm{g}}^{\text {Bulk }}+\frac{\hbar^{2} \alpha_{n l}^{2}}{2 \mathrm{r}^{2}}\left[\frac{1}{\mathrm{~m}_{\mathrm{e}}^{*}}+\frac{1}{\mathrm{~m}_{\mathrm{h}}^{*}}\right]-\frac{1.786 \mathrm{e}^{2}}{4 \pi \in_{\mathrm{o}} \in_{\mathrm{r}} \mathrm{r}}
$$

where, $\alpha_{\mathrm{nl}}$ is the dimensionless series that takes discrete values. For the lowest allowed interband transition $\alpha_{10}=\pi$ that is from $1 \mathrm{~s}$ hole to $1 \mathrm{~s}$ electron states (Equation (8)). The next values in this sequence, $\alpha_{11}=4.4934, \alpha_{12}=5.7635, \alpha_{20}=2 \pi$ etc. Using $\alpha_{11}$ the energy levels of second allowed transition were calculated and obtained values (3.73eV-2.6eV respectively for 0.08M TGA-capped QDs aliquots collected at 30 and 500 min reflux) well matches with the experimental values of the high band gap energy $(3.8 \mathrm{eV}-2.6 \mathrm{eV})$. Proving that the synthesized QDs are well confined with narrow size distribution and the transition show is from first two interband transitions. The Fig. $10 \mathrm{a}$ and $\mathrm{b}$ clearly depicts that as QDs size increases the band gap decreases, implies that as reflux time as increased size of QDs as grown, resulting in reduced quantum confinement and hence decreased band gap moving towards bulk band gap. Also, 0.08M concentration as large size QDs indicating that it is the preferred concentration then 0.04M to obtain a wide range of QDs. All the achieved QDs properties can be used in many applications, mainly its tunable band gap can be used in multi-layered stacked solar cells and space grade solar cells to achieve high efficiency, PL properties can be used in biological applications and LED. Moreover, QDs can also be used in traditional filters and detectors.

\section{Conclusions}

High-quality water soluble TGA stabilized CdTe Quantum dots have been synthesized successfully through a single injection hydrothermal method in complete ambient environment preventing oxidation of elemental Te powder as Tellurium precursors. The growth starts with fast nucleation and then follows with slow OR process, this mechanism and its effects on other properties are clearly analyzed. The XRD spectrum confirmed the synthesized QDs are CdTe in cubic zinc blend structure oriented along 111 planes. The QDs optical properties give insight into the importance of the concentration of the capping agent and its influence on other properties, due to the interaction between capping ligand and the core (CdTe) of QDs. Further, the optical spectrum studies confirm that 0.08M TGA concentration is strong enough in stabilizing the QDs and results in high QY, whereas, at low TGA concentration $(0.04 \mathrm{M})$ the capping was insufficient and leads to white turbulence and precipitation leading to low QY. Also, 0.08M TGA QDs had wide PL emission window covering most of the visible spectrum. The band gap of the synthesized QDs is well confined resulting multiple band energy and ranging from 3.8 to $1.97 \mathrm{eV}$. Hence, the proposed QDs synthesis protocol can be upscaled for industrial production and can clearly be used in the vast application of optoelectronic, photovoltaic and biological applications.

\section{Funding}

This research did not receive any specific grant from funding agencies in the public, commercial, or not-for-profit sectors.

\section{Acknowledgements}

The authors are thankful to research colleges of thin film solar cell lab for their support and Mr. Pradeep, IISc, Bangalore for helping in characterizations.

\section{References}

[1] L.E. Brus, Electron-electron and electron-hole interactions in small semiconductor crystallites: the size dependence of the lowest excited electronic state, J. Chem. Phys. 80 (1984) 4403, http://dx.doi.org/10.1063/1.447218. 
[2] V. Esch, B. Fluegel, G. Khitrova, H. Gibbs, K. Kang, S. Koch, L. Liu, S. Risbud, N. Peyghambarian, State filling, Coulomb, and trapping effects in the optical nonlinearity of CdTe quantum dots in glass, Phys. Rev. B 42 (1990) 7450-7453, http://dx.doi.org/10.1103/PhysRevB.42.7450.

[3] B. Jai Kumar, D. Sumanth Kumar, H.M. Mahesh, A facile single injection Hydrothermal method for the synthesis of thiol capped CdTe quantum dots as light harvesters, J. Lumin 178 (2016) 362-367, http://dx.doi.org/10.1016/j.jlumin.2016.06.012.

[4] A. Ruland, C. Schulz-Drost, V. Sgobba, D.M. Guldi, Enhancing photocurrent efficiencies by resonance energy transfer in CdTe quantum dot multilayers: towards rainbow solar cells, Adv. Mater. 23 (2011) 4573-4577, http://dx.doi.org/10.1002/adma.201101423.

[5] H. Wang, L. Sun, Y. Li, X. Fei, M. Sun, C. Zhang, Y. Li, Q. Yang, Layer-by-layer assembled $\mathrm{Fe}_{3} \mathrm{O}_{4} @$ C@CdTe core/shell microspheres as separable luminescent probe for sensitive sensing of $\mathrm{Cu}^{2+}$ ions, Langmuir 27 (2011) 11609-11615, http://dx.doi.org/10.1021/la202295b.

[6] V. Lesnyak, A. Wolf, A. Dubavik, L. Borchardt, S.V. Voitekhovich, N. Gaponik, S. Kaskel, A. Eychmüller, 3D assembly of semiconductor and metal nanocrystals: hybrid CdTe/Au structures with controlled content, J. Am. Chem. Soc. 133 (2011) 13413-13420, http://dx.doi.org/10.1021/ja202068s.

[7] E. Talgorn, M.a. De Vries, D.a. L., Photoconductivity enhancement in multilayers of CdSe and CdTe quantum dots, Society (2011) 1-8, http://dx.doi.org/ $10.1021 / \mathrm{nn} 2009134$.

[8] Y. Wang, Z. Tang, S. Tan, N.A. Kotov, Biological assembly of nanocircuit prototypes from protein-modified CdTe nanowires, Nano Lett. 5 (2005) 243-248, http://dx.doi.org/10.1021/nl0482682.

[9] W.R. Algar, A.J. Tavares, U.J. Krull, Beyond labels: a review of the application of quantum dots as integrated components of assays, bioprobes, and biosensors utilizing optical transduction, Anal. Chim. Acta 673 (2010) 1-25, http://dx.doi.org/10.1016/j.aca.2010.05.026.

[10] H. Kuang, Y. Zhao, W. Ma, L. Xu, L. Wang, C. Xu, Recent developments in analytical applications of quantum dots, TrAC Trends Anal. Chem. 30 (2011) 1620-1636, http://dx.doi.org/10.1016/j.trac.2011.04.022.

[11] M. Green, The nature of quantum dot capping ligands, J. Mater. Chem. 20 (2010) 5797-5809, http://dx.doi.org/10.1039/C0JM00007H.

[12] J. Liang, Y. Cheng, H. Han, Study on the interaction between bovine serum albumin and CdTe quantum dots with spectroscopic techniques, J. Mol. Struct. 892 (2008) 116-120, http://dx.doi.org/10.1016/j.molstruc.2008.05.005.

[13] Y. hai Zhang, H. shan Zhang, M. Ma, X. feng Guo, H. Wang, The influence of ligands on the preparation and optical properties of water-soluble CdTe quantum dots, Appl. Surf. Sci. 255 (2009) 4747-4753, http://dx.doi.org/10.1016/j.apsusc.2008.09.009.

[14] V. Swayambunathan, D. Hayes, K.H. Schmidt, Y.X. Liao, D. Meisel, Thiol surface complexation on growing cadmium sulfide clusters, J. Am. Chem. Soc. 112 (1990) 3831-3837, http://dx.doi.org/10.1021/ja00166a017.

[15] C. Frigerio, D.S.M. Ribeiro, S.S.M. Rodrigues, V.L.R.G. Abreu, J.A.C. Barbosa, J.A.V. Prior, K.L. Marques, J.L.M. Santos, Application of quantum dots as analytical tools in automated chemical analysis: a review, Anal. Chim. Acta 735 (2012) 9-22, http://dx.doi.org/10.1016/j.aca.2012.04.042.

[16] W.W. Yu, Y.a. Wang, X.G. Peng, Formation and stability of size-, shape-, and structure-controlled CdTe nanocrystals: ligand effects, Chem. Mater. (2003) 4300-4308, http://dx.doi.org/10.1021/cm034729t.

[17] D.V. Talapin, S. Haubold, A.L. Rogach, A. Kornowski, M. Haase, H. Weller, A novel organometallic synthesis of highly luminescent CdTe nanocrystals, J. Phys. Chem. B 105 (2001) 2260-2263, http://dx.doi.org/10.1021/jp003177o.

[18] Y. He, L.M. Sai, H.T. Lu, M. Hu, W.Y. Lai, Q.L. Fan, L.H. Wang, W. Huang, Microwave-assisted synthesis of water-dispersed CdTe nanocrystals with high luminescent efficiency and narrow size distribution, Chem. Mater. 19 (2007) 359-365, http://dx.doi.org/10.1021/cm061863f.

[19] H. Zhang, L. Wang, H. Xiong, L. Hu, B. Yang, W. Li, Hydrothermal synthesis for high-quality CDTe nanocrystals, Adv. Mater. 15 (2003) 1712-1715, http:// dx.doi.org/10.1002/adma.200305653.

[20] C. Wang, H. Zhang, J. Zhang, M. Li, H. Sun, B. Yang, Application of ultrasonic irradiation in aqueous synthesis of highly fluorescent CdTe/CdS core-shell nanocrystals, J. Phys. Chem. C 111 (2007) 2465-2469, http://dx.doi.org/10.1021/jp066601f.

[21] A.M. Kapitonov, A.P. Stupak, S.V. Gaponenko, E.P. Petrov, A.L. Rogach, A. Eychmüller, Luminescence properties of thiol-stabilized CdTe nanocrystals, J. Phys. Chem. B 103 (1999) 10109-10113, http://dx.doi.org/10.1021/jp9921809.

[22] C. Ge, M. Xu, J. Liu, J. Lei, H. Ju, Facile synthesis and application of highly luminescent CdTe quantum dots with an electrogenerated precursor, Chem. Commun. (2008) 450-452, http://dx.doi.org/10.1039/B714990E.

[23] J.-J. Shi, S. Wang, T.-T. He, E.S. Abdel-Halim, J.-J. Zhu, Sonoelectrochemical synthesis of water-soluble CdTe quantum dots, Ultrason. Sonochem. 21 (2014) 493-498, http://dx.doi.org/10.1016/j.ultsonch.2013.06.003.

[24] A.W. Zhao, G.W. Meng, L.D. Zhang, T. Gao, S.H. Sun, Y.T. Pang, Electrochemical synthesis of ordered CdTe nanowire arrays, Appl. Phys. A Mater. Sci. Process 76 (2003) 537-539, http://dx.doi.org/10.1007/s00339-002-1713-0.

[25] A.L. Rogach, T. Franzl, T.A. Klar, J. Feldmann, N. Gaponik, V. Lesnyak, A. Shavel, A. Eychmüller, Y.P. Rakovich, J.F. Donegan, Aqueous synthesis of thiolcapped CdTe Nanocrystals: state-of-the-art, J. Phys. Chem. C 111 (2007) 14628-14637, http://dx.doi.org/10.1021/jp072463y.

[26] J. Lee, A.O. Govorov, J. Dulka, N.A. Kotov, Bioconjugates of CdTe nanowires and Au nanoparticles: Plasmon-exciton interactions, luminescence enhancement, and collective effects, Nano Lett. 4 (2004) 2323-2330, http://dx.doi.org/10.1021/nl048669h.

[27] N. Gaponik, D.V. Talapin, A.L. Rogach, K. Hoppe, V. Shevchenko, A. Kornowski, A. Eychmller, H. Weller, E.V. Shevchenko, A. Eychmu, Thiol-capping of CdTe nanocrystals: an alternative to organometallic synthetic routes thiol-capping of CdTe nanocrystals: an alternative to organometallic synthetic routes, J. Phys. Chem. B (2002) 7177-7185, http://dx.doi.org/10.1021/jp025541k.

[28] Y. Zheng, S. Gao, J.Y. Ying, Synthesis and cell-imaging applications of glutathione-capped CdTe quantum dots, Adv. Mater. 19 (2007) 376-380, http:// dx.doi.org/10.1002/adma.200600342.

[29] Y. Liu, Wei Chen, A.G. Joly, Y. Wang, C. Pope, Y. Zhang, J.O. Bovin, P. Sherwood, Comparison of water-soluble CdTe nanoparticles synthesized in air and in nitrogen, J. Phys. Chem. B 110 (2006) 16992-17000, http://dx.doi.org/10.1021/jp063085k.

[30] Q. Wang, Y. Kuo, Y. Wang, G. Shin, C. Ruengruglikit, Q. Huang, Luminescent properties of water-soluble denatured bovine serum albumin-coated CdTe quantum dots, J. Phys. Chem. B 110 (2006) 16860-16866, http://dx.doi.org/10.1021/jp062279x.

[31] M. Gao, S. Kirstein, H. Möhwald, A.L. Rogach, A. Kornowski, A. Eychmüller, H. Weller, Strongly photoluminescent CdTe nanocrystals by proper surface modification, J. Phys. Chem. B 102 (1998) 8360-8363, http://dx.doi.org/10.1021/jp9823603.

[32] A.C. Nanoparticles, The influence of carboxyl groups on the photoluminescence the influence of carboxyl groups on the photoluminescence of mercaptocarboxylic, Solutions (2003) 8-13, http://dx.doi.org/10.1021/ip025910c.

[33] Q. Shang, H. Wang, H. Yu, G. Shan, R. Yan, Effect of phenylalanine on photoluminescence and stability of CdTe nanocrystals capped with thioglycolic acid, Colloids Surf. A Physicochem. Eng. Asp. 294 (2007) 86-91, http://dx.doi.org/10.1016/j.colsurfa.2006.07.058,

[34] W.L. Bragg, The dawn of X-ray crystallography, Proc. Camb. Philol. Soc. 17 (1913) 43-57.

[35] J. Tauc, R. Grigorovici, A. Vancu, Optical properties and electronic structure of amorphous germanium, Phys. Stat. Sol. 15 (1966) 627-637, http://dx.doi. org/10.1016/0025-5408(68)90023-8. 